V. van Kampen

R. Merget

T. Brüning

\title{
Berufliche Allergien gegen Xylanasen
}

\section{Occupational Allergies to Xylanases}

\section{Zusammenfassung}

Das Auftreten beruflich bedingter Allergien nach Exposition gegenüber Enzymstäuben ist seit längerem bekannt. So fiel das hohe Sensibilisierungspotenzial von Enzymen erstmals in den 60er-Jahren auf, als eine Reihe von beruflich bedingten Asthmaund Rhinitiserkrankungen in der Waschmittelproduktion auftraten. Wie die hier vorgestellte Auswertung von Literaturdaten zeigt, trifft dies auch auf die in der Industrie häufig eingesetzten Xylanasen zu. Diese zählen zu den Hemicellulasen und stammen in der Regel aus Schimmelpilzen. Durch eine größere Zahl von Kasuistiken und Querschnittstudien sind Fälle von spezifischer Überempfindlichkeit der Atemwege oder der Lunge durch Xylanasen gut belegt. Da Symptome, Hauttestergebnisse, der Nachweis spezifischer IgE-Antikörper und die Ergebnisse spezifischer Provokationstests in der Regel gut übereinstimmen, ist ein immunologischer Wirkmechanismus gesichert.

\section{Abstract}

The exposure against enzyme dusts have long been known to cause occupational allergies. In the $1960 \mathrm{~s}$ an increasing number of occupational allergies in the detergent industry were observed. In this context the high sensitization potential of enzyme dusts attracted attention. The present evaluation of literature data confirms that this is also true for xylanases. These frequently used industrial enzymes belong to the hemicellulases and are mostly of fungal origin. Several cases of specific airway sensitization caused by xylanases or other hemicellulases are verified by a number of case reports and cross sectional studies. As symptoms, results of skin prick tests, detection of specific IgE-antibodies and results of specific bronchoprovocation tests are consistent, an immunologic mechanism can be assumed.

\section{Einleitung}

Xylanasen bauen Xylan, einen holzigen, gummiartigen Schleimstoff (auch: Holzgummi) ab, der zusammen mit anderen Hemicellulosen (Glucane, Galactane, Mannane, Pentosane) und Cellulose die Polysaccharidbausteine pflanzlicher Zellwände darstellt.
Xylan besteht aus einer 1,4- $\beta$-verknüpften D-XylopyranosylHauptkette mit unterschiedlichen Substituenten. Es handelt sich um die in der Zellwand am häufigsten vorkommende Hemicellulose, die mehr als 30\% des Trockengewichts ausmachen [1]. Der Abbau von Xylan zum Monomer (Xylose) ist ein komplexer Prozess, an dem verschiedene xylanolytische Enzyme beteiligt 
sind, die unter dem Begriff Xylanasen zusammengefasst werden. Von besonderer Bedeutung sind die $\beta$-1,4-Endoxylanase (EC 3.2.1.8) und die $\beta$-Xylosidase (EC 3.2.1.37) [1].

Biotechnologisch werden Xylanasen in der Regel in Form von Kombinationspräparaten mit mehreren spezifisch wirkenden Enzymen eingesetzt. Aus diesem Grunde ist eine klare Abgrenzung zwischen den einzelnen Enzymen nicht immer möglich. Industrielle Verwendung finden Xylanasen vor allem als Backmittelenzyme zur Verbesserung der Teigeigenschaften (Maschinengängigkeit, Stabilität) und zur Produktoptimierung (Krustenstabilität, Volumen). Darüber hinaus werden sie in der Stärkeindustrie, bei der Spirituosenherstellung und in der Alkoholindustrie (Xylanasen schließen die Schleimstoffe der Getreide auf, die so für die Fermentation nutzbar werden), bei der Fruchtsaft- und Getränkeherstellung, als Futterzusatz (bessere Verwertung der Futtermittel) und in der Textil- und Papierindustrie eingesetzt. Xylanasen werden zunehmend mithilfe gentechnisch veränderter Mikroorganismen hergestellt. Die wichtigsten Xylanase-produzierenden Organismen sind Schimmelpilze der Gattungen Aspergillus, Penicillium und Trichoderma. Aufgrund der weiten Verbreitung von Xylan und der vielen Einsatzmöglichkeiten kommt den Xylanasen eine besondere Bedeutung unter den Hemicellulasen $\mathrm{zu}$.

\section{Literaturauswertung}

Mit Hilfe geeigneter Stichwörter in sinnvoller Kombination wurden die Abstracts infrage kommender Veröffentlichungen in einer Datenbank (MEDLINE) gefunden. Uns im Original zugängliche relevante Publikationen über Studien zur sensibilisierenden Wirkung von Xylanasen bzw. anderen Hemicellulasen wurden hinsichtlich der klinischen Daten ausgewertet. Erfasst wurden neben der Zahl der exponierten Personen die Prävalenzen der einzelnen Symptome bzw. Diagnosen. Darüber hinaus wurde festgehalten, mit welchen Methoden (Hauttest, Bestimmung spezifischer IgE-Antikörper, Provokationstest) und unter Verwendung welcher Testlösungen eine auf Xylanasen (Hemicellulasen) zurückzuführende Sensibilisierung diagnostiziert wurde.

\section{Ergebnisse}

Die Auswertung der Literatur zeigt, dass Xylanasen ebenso wie vielen anderen Enzymen, z.B. Subtilisinen [2] und Cellulasen [3], ein hohes Sensibilisierungspotenzial zukommt (Übersicht bei [4]). Neben der atemwegssensibilisierenden Wirkung wurden in einigen Fällen auch Hautsensibilisierungen durch xylanasehaltige Produkte beschrieben.

\section{Hautsensibilisierende Wirkung}

Bei 2 Beschäftigten traten nach 0,5- bzw. 4-jähriger Exposition gegen Xylanase zunächst allergische Reaktionen an der Haut in Form von Kontakturtikaria auf, denen später Rhinitis und Asthma folgten. Bei einem Patienten wurde ein 48-stündiger Epikutantest durchgeführt, der nach 48 und 96 Stunden ein deutlich positives Ergebnis auf 0,33; 1 und 3,3\% Xylanase in Vaseline zeigte, wobei die Reaktion direkt nach Abnahme der Testpflaster einen urtikariellen Anteil aufwies. 20 Kontrollpersonen zeigten keine
Reaktion auf die 3,3\%igen Zubereitungen. Im Pricktest reagierten beide Patienten positiv auf 0,001 bzw. 0,01\% Xylanase [5].

\section{Atemwegssensibilisierende Wirkung}

Einen zusammenfassenden Überblick über die Ergebnisse der Literaturauswertung hinsichtlich der atemwegssensibilisierenden Wirkung von Xylanasen bzw. Hemicellulasen gibt Tab. 1.

Alle 171 in einer retrospektiven Studie von 1992 bis 1997 untersuchten symptomatischen Bäcker klagten über Atemwegsbeschwerden, z.T. über Rhinitis und Konjunktivitis beim Umgang mit enzymhaltigen Backmitteln. Mittels Enzyme Allergo Sorbent Test (EAST) konnten in 18 (11\%) der 171 asservierten Seren spezifische IgE-Antikörper gegen Xylanase aus Aspergillus niger nachgewiesen werden. Mittels EAST-Inhibitionstest wurde die Spezifität der Antikörper überprüft. Zudem konnte mit Hilfe dieser Methode eine deutliche Kreuzreaktion (> 70\%) zwischen Xylanase und Cellulase ermittelt werden. Im Immunoblot wurde das am stärksten IgE-bindende Protein als $\beta$-Xylosidase (105 kDa) identifiziert [6].

124 Beschäftigte der enzymverarbeitenden Abteilung eines Chemiebetriebes wurden im Rahmen einer Querschnittstudie untersucht. 61 Personen waren in der Herstellung der Enzyme aus Aspergillus niger, A. oryzae und Bacillus subtilis, 42 Personen in der Weiterverarbeitung von Enzymen zu Wasch- und Backmitteln und 21 Personen in Laboratorien beschäftigt. Luftstaubmessungen und anschließende Enzymaktivitätsbestimmungen ergaben für Xylanase im Bereich der Enzymherstellung einen Wert von $3,3 \mu \mathrm{g} / \mathrm{m}^{3}$, im Bereich der Weiterverarbeitung einen Wert von $0,1 \pm 0,1 \mu \mathrm{g} / \mathrm{m}^{3}$. Insgesamt klagten 70 Beschäftigte (56,5\%) über arbeitsplatzbezogene Beschwerden (Rhinitis, Konjunktivitis, Husten und Luftnot). Diese Beschwerden wurden bei der Enzymherstellung und -weiterverarbeitung deutlich häufiger angegeben als in den Laboratorien. Der Pricktest mit wässrigem Xylanaseextrakt (keine weiteren Angaben (k.w.A.)) ergab bei 12,1\% (Aspergillus-Xylanase) bzw. 8,9\% (B. subtilis-Xylanase) der Probanden ein positives Ergebnis. Spezifische IgE-Antikörper gegen Enzyme fanden sich häufiger bei Beschäftigten mit arbeitsplatzbezogenen und arbeitsplatzunabhängigen Atemwegsbeschwerden als in der beschwerdefreien Gruppe (k. w.A.) [7].

In einer Querschnittuntersuchung unter 49 Enzymexponierten wurden arbeitsplatzbezogene Beschwerden in der Gruppe der Hochexponierten ( $n=8$; Rhinitis $100 \%$, Konjunktivitis $87 \%$, Bronchitis $63 \%$ ) häufiger angegeben als in der Gruppe der Niedrigexponierten $(n=41)$. Das in der Anamnese auffällige Teilkollektiv $(\mathrm{n}=32$ ) wurde im Pricktest mit Endoxylanase und Phytase untersucht (k.w. A). Bei zwei Personen zeigte sich dabei eine deutlich positive Reaktion, die in einem Fall so stark ausfiel, dass eine zusätzliche nasale Provokation mit Xylanase nicht möglich war. Im anderen Fall konnte die Xylanasesensibilisierung durch eine nasale Provokation bestätigt werden. Insgesamt lag die Sensibilisierungsrate im Gesamtkollektiv gegen Xylanase und Phytase bei $24 \%$ [8].

Zwei finnische Beschäftigte biotechnologischer Laboratorien litten nach beruflicher Xylanase- und Cellulaseexposition unter Atemwegsbeschwerden und Hautsymptomen. Sie wurden im Pricktest mit 0,01 oder 0,001\% Xylanase in Coca-Lösung/Glycerin 
Tab. 1 Klinische Untersuchungen zur atemwegssensibilisierenden Wirkung von Xylanasen bzw. Hemicellulasen

\begin{tabular}{|c|c|c|c|c|c|}
\hline \multirow[t]{2}{*}{ Studie } & \multirow{2}{*}{$\begin{array}{l}\text { Zahl der Unter- } \\
\text { suchten }\end{array}$} & \multirow{2}{*}{$\begin{array}{l}\text { arbeitsplatzbezogene } \\
\text { Symptome bzw. klinische } \\
\text { Diagnosen }\end{array}$} & \multicolumn{3}{|l|}{ Nachweismethode } \\
\hline & & & $\begin{array}{l}\text { Pricktest } \\
\text { (Testkonz.) }\end{array}$ & $\begin{array}{l}\text { spezif. IgE-Antikörper } \\
\text { (Methode) }\end{array}$ & $\begin{array}{l}\text { bronchiale Provokation } \\
\text { (Testkonz.) }\end{array}$ \\
\hline 5 & 2 & $\begin{array}{l}\text { bei beiden Atemwegsbeschwerden, } \\
\text { Hautsymptome }\end{array}$ & $\begin{array}{l}\text { positiv bei } 2 / 2(0,01 \% \text { oder } \\
0,001 \% \text { in Coca-Lösung/ } \\
\text { Glycerin, } 1: 1)\end{array}$ & positiv bei 2/2 (RAST ) & - \\
\hline 6 & 171 & $\begin{array}{l}\text { bei allen Rhinitis, Konjunktivitis und/oder } \\
\text { Atemwegsbeschwerden }\end{array}$ & - & positiv bei 18/171 (EAST) & - \\
\hline 7 & 124 & $\begin{array}{l}70(56 \%) \text { Rhinits, Konjunktivitis, } \\
\text { Husten und/oder Luftnot }\end{array}$ & $\begin{array}{l}\text { positiv bei } 12,1 \% \text { (Aspergil- } \\
\text { lus.) bzw. } 8,9 \% \text { (B. subtilis) } \\
\text { (wässriger Extrakt, k. w. A.) }\end{array}$ & $\begin{array}{l}\text { spez. Antikörper häufiger } \\
\text { bei Symptomatischen als } \\
\text { bei Beschwerdefreien } \\
\text { (k.w. A.) }\end{array}$ & - \\
\hline 8 & 49 & $\begin{array}{l}\text { unter Hochexponierten } n=8 \text { : Rhinitis } \\
100 \% \text {, Konjunktivitis } 87 \% \text {, Bronchitis } \\
63 \% \text {. } \\
\text { unter Niedrig-exponierten } n=41 \text { : Symp- } \\
\text { tome seltener }\end{array}$ & positiv bei 2/32 (k.w. A.) & - & npositiv 1/1 (k.w.A.) \\
\hline 9 & 1 & Rhinitis, Konjunktivitis, Kurzatmigkeit & positiv (k. w. A.) & positiv (EAST) & positiv $(1,056 \mu \mathrm{g})$ \\
\hline 10 & 1 & Rhinitis, Konjunktivitis, Husten, Luftnot & positiv $(0,1 \mathrm{mg} / \mathrm{ml})$ & positiv (EAST) & positiv (ca. 0,5 $\mu \mathrm{g}$ ) \\
\hline 11 & 365 & $\begin{array}{l}57(16 \%) \text { Rhinitis, Konjunktivitis, Atem- } \\
\text { wegsbeschwerden und/oder Hautsymp- } \\
\text { tome }\end{array}$ & $\begin{array}{l}\text { alle negativ (Proteinkonz.: } \\
50 \mu \mathrm{g} \text { Xylanase/ml Coca- } \\
\text { Glycerin-Lösung) }\end{array}$ & alle negativ (RAST) & - \\
\hline 12 & 140 & $\begin{array}{l}\text { 16\% Rhinitis, } \\
4 \% \text { Beschwerden unterer Atemtrakt, } \\
19 \% \text { Beschwerden oberer Atemtrakt }\end{array}$ & $\begin{array}{l}\text { positiv bei } 7 / 140 \text { auf } \\
\text { Xylanase, Cellulase oder } \\
\beta \text {-Glucanase (Proteinkon- } \\
\text { zentration: } 100 \mu \mathrm{g} / \mathrm{ml})\end{array}$ & - & - \\
\hline 13 & 135 & k.w. A & - & $\begin{array}{l}\text { positiv bei } 8 / 135 \text { (RAST) mit } \\
\text { Enzymmischung (Xylanase, } \\
\text { Hemicellulase, Cellulase) }\end{array}$ & - \\
\hline *14 & 140 & $\begin{array}{l}\text { bei allen Rhinitis, Konjunktivitis und/oder } \\
\text { Asthma }\end{array}$ & - & positiv bei $11 / 140$ (RAST) & - \\
\hline *15 & 42 & $\begin{array}{l}\text { bei allen Rhinitis, Konjunktivitis, Asthma, } \\
\text { Hautbeschwerden }\end{array}$ & $\begin{array}{l}\text { positiv bei } 4 / 29 \text { (max. } \\
10 \mathrm{mg} / \mathrm{ml} 0,9 \% \mathrm{NaCl}, 0,4 \% \\
\text { Phenol, } 50 \% \text { Glycerin) }\end{array}$ & positiv bei 2/29 (EAST) & $\begin{array}{l}\text { positiv bei } 2 / 29 \text { (max. } \\
1 \mathrm{mg} / \mathrm{ml}(=0,36 \mathrm{mg} \\
\text { Protein } / \mathrm{ml} \text { ) in } 0,9 \% \\
\mathrm{NaCl}, 0,4 \% \text { Phenol) }\end{array}$ \\
\hline
\end{tabular}

k. w. A keine weiteren Angaben. ${ }^{n}$ nasale Provokation, ${ }^{*}$ Hemicellulasen (ohne nähere Angaben)

(1:1) untersucht und zeigten eine deutlich positive Reaktion; 20 nichtexponierte Kontrollpersonen reagierten nicht. Mittels Radio Allergo Sorbent Test (RAST) konnten im Serum beider Patienten Xylanase-spezifische IgE-Antikörper nachgewiesen werden. Die Spezifität der Antikörper wurde mittels RAST-Inhibitionstest überprüft. Mit Hilfe dieser Methode wurde eine Kreuzreaktion (>90\%) zwischen Xylanase und der Cellulase ermittelt. Bei beiden Patienten wurde aufgrund der Symptome, eines hyperreaktiven Bronchialsystems und aufgrund des Abfalls des exspiratorischen Spitzenflusses (PEF) bei beruflicher Exposition ein beruflich bedingtes Asthma diagnostiziert [5].

Eine Kasuistik beschreibt den Fall eines 35-jährigen Bäckers, der seit 3 Jahren über arbeitsplatzbezogene Symptome (Rhinitis, Konjunktivitis, Kurzatmigkeit) klagte, die während des Urlaubs verschwanden. Im Pricktest reagierte er deutlich auf Xylanase (Quaddeldurchmesser: $16 \mathrm{~mm}$ ) und schwächer auf Cellulase (Quaddeldurchmesser: $6 \mathrm{~mm}$ ), die beide aus Aspergillus niger stammten. Mittels EAST waren Xylanase-spezifische IgE-Antikörper nachweisbar. Die Spezifität der Antikörper konnte im EASTInhibitionstest bestätigt werden. Darüber hinaus wurde eine Kreuzreaktivität zwischen Xylanase und Cellulase gefunden. Die
Xylanasesensibilisierung wurde im bronchialen Provokationstest bestätigt. Nach schrittweiser Erhöhung der Konzentration kam es bei einer Xylanasekonzentration von $50 \mu \mathrm{g} / \mathrm{ml}$ (kumulative Dosis: 1,056 $\mu \mathrm{g}$ ) zu Kurzatmigkeit bei Verdopplung des spezifischen Atemwegswiderstandes. Nach gelelektrophoretischer Auftrennung des Xylanaseextraktes fanden sich im Immunoblot verschiedene IgE-bindende Proteine mit Molekulargewichten unterhalb von 100 kDa mit einer Hauptbande um 67 kDa [9].

In einer weiteren Kasuistik wird der Fall eines 26-jährigen Bäckers beschrieben, der seit 3 Jahren unter arbeitsplatzbezogenen Beschwerden (Rhinokonjunktivitis, Husten, Kurzatmigkeit) litt. Der Pricktest mit den Enzymen Xylanase $(0,1 \mathrm{mg} / \mathrm{ml})$ und Cellulase $(1 \mathrm{mg} / \mathrm{ml})$ aus Aspergillus niger sowie $\alpha$-Amylase aus A. oryzae $(1 \mathrm{mg} / \mathrm{ml})$ fiel positiv aus, mit der stärksten Reaktion auf Xylanase. Mittels EAST waren Xylanase-spezifische IgE-Antikörper nachweisbar. Die Xylanasesensibilisierung wurde im bronchialen Provokationstest bestätigt. Nach Applikation von ca. 0,5 $\mu \mathrm{g}$ Xylanase kam es zu einer Verdopplung des spezifischen Atemwegswiderstandes. Bei zwei nichtexponierten asthmatischen Kontrollen verlief die bronchiale Provokation mit Xylanase negativ [10]. 
In einer 1996 publizierten finnischen Querschnittstudie wurden 365 Mitarbeiter von 5 Backbetrieben und einer Mühle hinsichtlich berufsbedingter Enzymallergien untersucht. Arbeitsplatzbezogene Symptome, zumeist Rhinitis, seltener Atemwegsbeschwerden, Konjunktivitis oder Urtikaria gaben 57 (16\%) der Beschäftigten an. Weder mittels Pricktest (50 $\mu \mathrm{g}$ Xylanase/ml in Coca-Glycerin-Lösung) noch mittels RAST konnte eine XylanaseSensibilisierung nachgewiesen werden [11].

In einer späteren Studie bestimmten Vanhanen u. Mitarb. [12] in der Raumluft von 4 Tierfutterfabriken die Xylanasekonzentrationen mithilfe einer immunologischen Methode, deren Nachweisgrenze bei $0,7 \mathrm{ng} / \mathrm{m}^{3}$ lag. Dabei ergaben sich Messwerte zwischen 0,8 und $16 \mathrm{ng} / \mathrm{m}^{3}$. Die Mittelwerte in den 4 Fabriken lagen zwischen 3,1 und 6,6 $\mathrm{ng} / \mathrm{m}^{3}$. Von insgesamt 140 exponierten Mitarbeitern, die teilweise über Rhinitis und Atemwegsbeschwerden klagten, reagierten 7 im Pricktest auf Xylanase, Cellulase oder $\beta$-Glucanase (jeweils $100 \mu \mathrm{g}$ Enzym/ml).

135 asservierte Seren aus einer früheren Querschnittstudie unter schottischen Bäckern wurden mittels RAST hinsichtlich ihres Gehaltes an IgE-Antikörpern gegen eine Enzymmischung (Xylanase, Cellulase, Hemicellulase) und $\alpha$-Amylase untersucht. In 8 Seren (6\%) waren spezifische IgE-Antikörper gegen die Enzymmischung und in 16 Seren (12\%) IgE-Antikörper gegen $\alpha$-Amylase nachweisbar. Eine Differenzierung der Ergebnisse mit der Enzymmischung nach den einzelnen darin enthaltenen Enzymen erfolgte nicht [13].

\section{Hemicellulasen}

In einer Studie wurden 140 Personen untersucht, die mindestens 6 Monate in einem Backbetrieb tätig waren und über berufsbedingte Beschwerden wie Asthma, Rhinitis und/oder Konjunktivitis klagten. Säulenchromatographisch gereinigte Enzym-Rohextrakte wurden zur Bestimmung spezifischer IgE-Antikörper mittels RAST verwendet. $8 \%$ der Untersuchten (11/140) wiesen eine Sensibilisierung gegenüber einer nicht näher beschriebenen Aspergillus-Hemicellulase auf, die auch Cellulaseaktivität aufwies. Mittels Immunoblot unter Verwendung positiver Seren wurden die IgE-bindenden Proteine analysiert. Die Hauptbande des Hemicellulaseextrakts hatte ein Molekulargewicht $>90 \mathrm{kDa}$ [14]

Bei 42 enzymexponierten Beschäftigten eines Chemiebetriebs, die alle unter arbeitsplatzbezogenen Beschwerden litten (Rhinitis, Konjunktivitis, Asthma, Hautbeschwerden) wurden insgesamt 82 bronchiale Provokationstests mit 9 verschiedenen Enzymen durchgeführt. 2 von 29 (7\%) Provokationen mit Hemicellulase (k.w.A.) in aufsteigender Konzentration $\left(10^{-4}\right.$ bis $5 \mathrm{mg} / \mathrm{ml}$ physiologischer Kochsalzlösung, 0,4\% Phenol) ergaben ein positives Ergebnis, was definiert war als 50\%iger Abfall der spezifischen Atemwegsleitfähigkeit $\left(\mathrm{sG}_{\mathrm{aw}}\right)$ bei einer Hemicellulasekonzentration unterhalb von $1 \mathrm{mg} / \mathrm{ml}$ (entspricht $0,36 \mathrm{mg}$ Protein/ $\mathrm{ml}$ ). Mittels EAST konnten ebenfalls bei 2 von 29 Personen Hemicellulase-spezifische IgE-Antikörper detektiert werden. Im Pricktest mit $(1,10,20,50,100 \mathrm{mg}$ Hemicellulase/ml physiologischer Kochsalzlösung, 0,4\% Phenol, 50\% Glycerin) zeigten 4 Personen ein positives Ergebnis (Quaddelgröße entsprechend der Histaminquaddel bei einer Enzymkonzentration $<10 \mathrm{mg} / \mathrm{ml}$ ) [15]

\section{Schllussfolgerung}

Durch eine größere Zahl von Studien sind Fälle von spezifischer Überempfindlichkeit der Atemwege oder der Lunge durch die in der Regel aus Pilzen stammenden Xylanasen gut belegt. Da Symptome, Hauttestergebnisse, der Nachweis spezifischer IgEAntikörper und die Ergebnisse spezifischer Provokationsteste in der Regel gut übereinstimmen, ist ein immunologischer Wirkmechanismus im Sinne einer IgE-vermittelten Allergie gesichert. Außerdem sind auch urtikarielle Sofortreaktionen der Haut durch Kontakt mit Xylanasen möglich. Eindeutige Hinweise auf eine zellvermittelte kontaktallergene Wirkung liegen nicht vor.

\section{Literatur}

${ }^{1}$ Sunna A, Antranikian G. Xylanolytic enzymes from fungi and bacteria. Crit Rev Biotechnol 1997; 17: 39-67

${ }^{2}$ Kampen V van, Merget R. Berufliche Atemwegssensibilisierungen durch Subtilisine. Pneumologie 2002; 56: 182-186

${ }^{3}$ Kampen V van, Lessmann H, Brüning T et al. Berufliche Allergien gegen Cellulasen. Pneumologie 2003; 57: 388-391

${ }^{4}$ Baur X, Sander I, Posch A et al. Baker's asthma due to the enzyme xylanase - a new occupational allergen. Clin Exp Allergy 1998; 28: $1591-1593$

${ }^{5}$ Tarvainen K, Kanerva L, Tupasela O et al. Allergy from cellulase and xylanase enzymes. Clin Exp Allergy 1991; 21: 609-615

${ }^{6}$ Sander I, Raulf-Heimsoth M, Siethoff C et al. Allergy to Aspergillus-derived enzymes in the baking industry: identification of beta-xylosidase from Aspergillus niger as a new allergen (Asp n 14). J Allergy Clin Immunol 1998; 102: 256-264

${ }^{7}$ Bolm-Audorff U, Bienfait G, Hiltl G et al. Atemwegsallergien bei Enzym-exponierten Chemiearbeitern. In: Schuckmann F. (Hrsg.). Verhandlungen der Deutschen Gesellschaft für Arbeitsmedizin, A.W. Stuttgart: Gentner, 1991: 201 - 205

${ }^{8}$ Straßburger K, Bossert J, Baur X et al. Sensibilisierung durch die Enzyme Phytase und Xylanase. Dokumentationsband der 38. Jahrestagung der DGAUM. 1998: 525-527

${ }^{9}$ Merget R, Sander I, Raulf-Heimsoth M et al. Baker's asthma due to xylanase and cellulase without sensitization to alpha-amylase and only weak sensitization to flour. Int Arch Allergy Immunol 2001; 124: $502-505$

${ }^{10}$ Baur X, Sander I, Posch A et al. Baker's asthma due to the enzyme xylanase - a new occupational allergen. Clin Exp Allergy 1998; 28: $1591-1593$

${ }^{11}$ Vanhanen M, Tuomi T, Hokkanen $\mathrm{H}$ et al. Enzyme exposure and enzyme sensitisation in the baking industry. Occup Environ Med 1996; 53: $670-676$

12 Vanhanen M, Tuomi T, Tiikkainen U et al. Sensitisation to enzymes in the animal feed industry. Occup Environ Med 2001; 58: 119-123

${ }^{13}$ Elms J, Fishwick D, Walker J et al. Prevalence of sensitisation to cellulase and xylanase in bakery workers. Occup Environ Med 2003; 60: $802-804$

${ }^{14}$ Baur X, Sauer W, Weiss W. Baking additives as new allergens in baker's asthma. Respiration 1988; 54: 70-72

${ }^{15}$ Merget R, Stollfuss J, Wiewrodt R et al. Diagnostic tests in enzyme allergy. J Allergy Clin Immunol 1993; 92: $264-277$

\section{Bereits publizierte Beiträge zu dieser Serie:}

${ }^{1}$ Kühlschmierstoffe. Pneumologie 2003; 57: 212-221

2 Getreidestaub. Pneumologie 2003; 57: 335 - 339

${ }^{3}$ Cellulasen. Pneumologie 2003; 57: $388-391$

${ }^{4}$ Isocyanate. Pneumologie 2003; 57: 526-531

5 Platinsalze. Pneumologie 2003; 57: 606-611

${ }^{6}$ Nickel. Pneumologie 2003; 57: 667-670 